\title{
Advanced Numerical Methods in Applied Sciences
}

\author{
Luigi Brugnano $1, *\left(\mathbb{C}\right.$ and Felice Iavernaro ${ }^{2, *}$ \\ 1 Dipartimento di Matematica e Informatica “U. Dini”, Università di Firenze, Viale Morgagni 67/A, \\ 50134 Firenze, Italy \\ 2 Dipartimento di Matematica, Università di Bari, Via Orabona 4, 70125 Bari, Italy \\ * Correspondence: luigi.brugnano@unifi.it (L.B.); felice.iavernaro@uniba.it (F.I.)
}

Received: 25 January 2019; Accepted: 29 January 2019; Published: 31 January 2019

Abstract: The use of scientific computing tools is, nowadays, customary for solving problems in Applied Sciences at several levels of complexity. The great need for reliable software in the scientific community conveys a continuous stimulus to develop new and more performing numerical methods which are able to grasp the particular features of the problem at hand. This has been the case for many different settings of numerical analysis, and this Special Issue aims at covering some important developments in various areas of application.

Keywords: numerical analysis; numerical methods; scientific computing

\section{Special Issue Overview}

The special issue contains 15 contributions covering a number of areas of application in Numerical Analysis and Scientific Computing, which we can summarize as follows:

1. Numerical Linear Algebra [1-3];

2. Numerical solution of differential equations [4-10];

3. Geometric integration [11,12];

4. Computer graphics [13];

5. Optimization $[14,15]$.

Below, we highlight the main results of the papers.

\subsection{Numerical Linear Algebra}

In [1], the authors study the generalized Schur algorithm (GSA), which allows to compute well-known matrix decompositions, such as the $Q R$ and $L U$ factorizations. In particular, they use the GSA to obtain new theoretical insights on the bounds of the entries of the matrix $R$ in the $Q R$ factorization of some structured matrices, with related applications.

In [2], the author deals with the definition of limited memory preconditioners for symmetric and positive definite matrices. The existing connections with similar preconditioners are also discussed, along with its efficient implementaion. Extensive numerical tests are reported.

The authors of [3] discuss block-generalized, locally Toeplitz sequences which arise, e.g., from the discretization of many kinds of differential equations. The theoretical framework is then recalled, also completing previous results from the same authors, and a number of examples derived from the numerical solution of differential equations are worked out.

\subsection{Numerical Solution of Differential Equations}

The author of [4], who pioneered the order analysis of Runge-Kutta methods based on the theory of trees, introduces here the more general concept of stump. Stumps are then applied to the analysis 
of B-series, and used to study the order of Runge-Kutta methods when applied to non-autonomous scalar problems.

In [5], the authors review recent findings on the use of collocation methods for numerically solving Volterra integral and integro-differential equations. Both one-step and multi-step methods are considered, studying their convergence and providing comparisons in terms of efficiency and accuracy.

The authors in [6] study systems of fractional differential equations, in which different equations may have a different fractional time derivative at the left-hand side term of the equation. The linear case is completely worked out, providing a theory which collapses to the well-known Mittag-Leffler solution in the case where the indices are the same.

Fractional differential equations are also studied in [7], where a numerical method based on B-splines is proposed for their solution. In particular, the fractional diffusion problem is considered, and its numerical solution worked out.

Stochastic differential equations are considered in [8], where the authors review stability issues related to stochastic ordinary and Volterra integral equations. Two-step methods are then considered for the numerical solution in the ordinary case, and the $\theta$ method in the case of Volterra equations.

The numerical solution of Black-Scholes-type partial differential equations is studied in [9], where the authors provide a numerical method, and a related Matlab ${ }^{\circledR}$ code, for pricing some kinds of Asian options.

Arbitrarily high-order schemes using derivatives discontinuous Galerkin (ADER-DG) finite element methods are studied in [10]. The proposed methods are applicable to a wide class of nonlinear systems of partial differential equations, and are aimed at efficiently scaling on massively parallel supercomputers, as is testified by the numerical tests.

\subsection{Geometric Integration}

In [11] the authors study a class of A-stable, symmetric, one-step Hermite-Obreshkov methods previously introduced by other authors, which are here proved to be conjugate-symplectic. Moreover, a new and efficient implementation of the corresponding continuous spline extension is introduced. Numerical tests on some Hamiltonian problems are reported.

The authors in [12] study the use of the so-called Line Integral Methods for numerically solving conservative problems. In particular, energy-conserving methods for Hamiltonian problems are reviewed, with a number of extensions to related problems, such as constrained Hamiltonian problems, highly-oscillatory problems, and Hamiltonian partial differential equations.

\subsection{Computer Graphics}

In [13], the authors study the efficient construction of (truncated) hierarchical B-splines. In particular, hierarchical refinement strategies are considered, to be used within the framework of the so-called isogeometric analysis for numerically solving partial differential equations. The theoretical properties of the refinement algorithms and the resulting meshes are thoroughly analyzed and presented together with extensive numerical testing.

\subsection{Optimization}

In [14], the authors describe a two-step procedure for solving the so-called low-rank matrix completion problem. In the first step, a one-dimensional optimization problem, which depends on a scalar parameter, is solved. In the second step, the same functional, now depending on a matrix, is minimized. This latter minimization is achieved by solving a related matrix ODE.

The authors of [15] study the so-called problem of histogram specification, one of the main important tools in image processing. In particular, they propose a convex model that can include additional constraints based on different applications in edge-preserving smoothing. The convexity of the model allows to compute the output efficiently by the Fast Iterative Shrinkage-Thresholding Algorithm or the Alternating Direction Method of Multipliers. 
Conflicts of Interest: The authors declare no conflict of interest.

\section{References}

1. Laudadio, T.; Matronardi, N.; van Dooren, P. The Generalized Schur Algorithm and Some Applications. Axioms 2018, 7, 81. [CrossRef]

2. Morini, B. On Partial Cholesky Factorization and a Variant of Quasi-Newton Preconditioners for Symmetric Positive Definite Matrices. Axioms 2018, 7, 44. [CrossRef]

3. Garoni, C.; Mazza, M.; Serra-Capizzano, S. Block Generalized Locally Toeplitz Sequences: From the Theory to the Applications. Axioms 2018, 7, 49. [CrossRef]

4. Butcher, J.C. Trees, Stumps, and Applications. Axioms 2018, 7, 52. [CrossRef]

5. Cardone, A.; Conte, D.; D'Ambrosio, R.; Paternoster, B. Collocation Methods for Volterra Integral and Integro-Differential Equations: A Review. Axioms 2018, 7, 45. [CrossRef]

6. Burrage, K.; Burrage, P.; Turner, I.; Zheng, F. On the Analysis of Mixed-Index Time Fractional Differential Equation Systems. Axioms 2018, 7, 25. [CrossRef]

7. Pitolli, F. Optimal B-Spline Bases for the Numerical Solution of Fractional Differential Problems. Axioms 2018, 7, 46. [CrossRef]

8. Cardone, A.; Conte, D.; D'Ambrosio, R.; Paternoster, B. Stability Issues for Selected Stochastic Evolutionary Problems: A Review. Axioms 2018, 7, 91. [CrossRef]

9. Aimi, A.; Diazzi, L.; Guardasoni, C. Efficient BEM-Based Algorithm for Pricing Floating Strike Asian Barrier Options (with MATLAB ${ }^{\circledR}$ Code). Axioms 2018, 7, 40. [CrossRef]

10. Dumbser, M.; Fambri, F.; Bader, M.T.M.; Weinzierl, T. Efficient Implementation of ADER Discontinuous Galerkin Schemes for a Scalable Hyperbolic PDE Engine. Axioms 2018, 7, 63. [CrossRef]

11. Mazzia, F.; Sestini, A. On a Class of Conjugate Symplectic Hermite-Obreshkov One-Step Methods with Continuous Spline Extension. Axioms 2018, 7, 58. [CrossRef]

12. Brugnano, L.; Iavernaro, F. Line Integral Solution of Differential Problems. Axioms 2018, 7, 36. [CrossRef]

13. Bracco, C.; Giannelli, C.; Vásquez, R. Refinement Algorithms for Adaptive Isogeometric Methods with Hierarchical Splines. Axioms 2018, 7, 43. [CrossRef]

14. Scalone, C.; Guglielmi, N. A Gradient System for Low Rank Matrix Completion. Axioms 2018, 7, 51. [CrossRef]

15. Chan, K.C.K.; Chan, R.H.; Nikolova, M. A Convex Model for Edge-Histogram Specification with Applications to Edge-Preserving Smoothing. Axioms 2018, 7, 53. [CrossRef]

(C) 2019 by the authors. Licensee MDPI, Basel, Switzerland. This article is an open access article distributed under the terms and conditions of the Creative Commons Attribution (CC BY) license (http://creativecommons.org/licenses/by/4.0/). 